\title{
A Framework of e-based Quality Management for Distributed Manufacturing System
}

\author{
Iraj Mahdavi \\ Mazandaran University of Science and Technology \\ E-Mail: irajarash@rediffmail.com \\ Babak Shirazi \\ Mazandaran University of Science and Technology \\ E-mail: shirazi_b@yahoo.com \\ Namjae Cho \\ School of Business, Hanyang University \\ E-mail: njcho@hanyang.ac.kr
}

\begin{abstract}
Advanced manufacturing systems need to be developed for an enterprise to survive in the increasingly competitive global market. Statistical e-based quality control approach combines statistical quality analyses and reporting capabilities with web technology to deliver process optimization solutions. In this paper we develop a mathematical structured profile for statistical e-based quality to provide the capacity to access required data anywhere. It helps enterprises to develop customized quality information systems, create and distribute reports via the internet, and to provide real-time display of quality profiles for processes monitoring. Quality engineers and managers have been dependent on IS departments to secure access to such data. Statistical e-based quality profile is designed to bridge the gap between raw data and genuine quality improvement efforts by providing a powerful web-based solution for real-time quality process.
\end{abstract}

Keywords: Distributed Manufacturing System, E-Based Quality Profile, Real-Time Process 


\section{INTRODUCTION}

The global diffusion of advanced manufacturing systems naturally resulted in the needs for distant product development, which in turn requires the provision of production information and knowledge on the place, time and configuration across product life cycle. In response to this need the research community has come with a solution called distributed manufacturing system (DMS), which is defined as "an Internet based computational architecture that supports the sharing and transfer of knowledge and information about the product and manufacturing process amongst geographically distributed companies to support right engineering decisions in e-based environment''(Rodriguez and Al-Ashaab, 2002).

A number of research initiatives related to e-based manufacturing systems have been undertaken by several authors. Information models and engineering applications are integrated to form a framework in a structured and transparent manner using communication protocols shared among elements of the system (Molina et al., 1995). The traditional manufacturing control systems have little capacity to adapt and react to the dynamic changes in environment. For this reason, new approaches are called for to provide the capability to adapt to changes without external interventions (Leitao and Restivo, 2000). The emergent manufacturing control architectures should use the multiagent technology to support the development of autonomous and adaptive control architectures. Nowadays, we also observe a trend for a high level of product customization to fulfill market demands, shifting the manufacturing paradigm from the era of mass production to the era of mass customization. The major requirements that emergent manufacturing systems should comprise include: enterprise integration, distributed organizational architecture, heterogeneous environments, and fault tolerant integration of human elements with the cooperative open and dynamic structure of modern organization. The network-type operation and processing further facilitates the adoption of concurrent systems in distributed computing environments. Significant changes have been made to enterprise strategies and manufacturing paradigms, particularly for companies working together in the global marketplace. Jagdev and Thoben (2001) recognize three types of enterprise collaboration: supply chain (SC), extended enterprise and virtual enterprise. Information and Communication Technologies (ICT) have stimulated the emergence and evolvement of various enterprise collaborations. A virtual enterprise is a network of independent organizations that jointly form an entity committed to provide a products or services. From the customer's 
perspective, as far as that product/service is concerned, these independent organizations, for all practical and operational purposes, virtually act as a single entity/enterprise (Hao et al., 2005).

In this paper we review the concept of advanced manufacturing, and also we study the structure of quality management for a DMS. The goal of this paper is to improve the quality of production and reduce loss through systematic tracking and the use of information as continuous feed-back to production lines

\section{ADVANCED MANUFACTURING SYSTEM}

The advanced manufacturing environment integrates a wide span of components of a factory as shown in Figure1. Integrated manufacturing systems span from a single equipment automation system to more sophisticated systems such as Manufacturing Execution System (MES), Yield Management System (YMS), Equipment Engineering Capability (EEC) and Enterprise Resource Planning (ERP) (Su et al., 2002).

One of the major concerns for a manufacturing company at present is to obtain product consistency. Naturally the importance of the quality process is highlighted. Quality management practices focus on reducing process variance, which has a direct impact on supply chain performance including inventory and lead time (Flynn et al., 1995).

If process variance is reduced, there is a less need for safety stock and cycle stock (Flynn and Flynn, 2005). The quality management problem has many different formulations while a set of core characteristics can be identified (Dean and Snell, 1991; Anderson et al. 1994; Mehra et al., 2001).

A manufacturing execution system shares its wealth of information with other parts inside a company as well as with key customers and suppliers. Such an extensive sharing of information leads to an increased level of quality assurance and visibility into operations. 


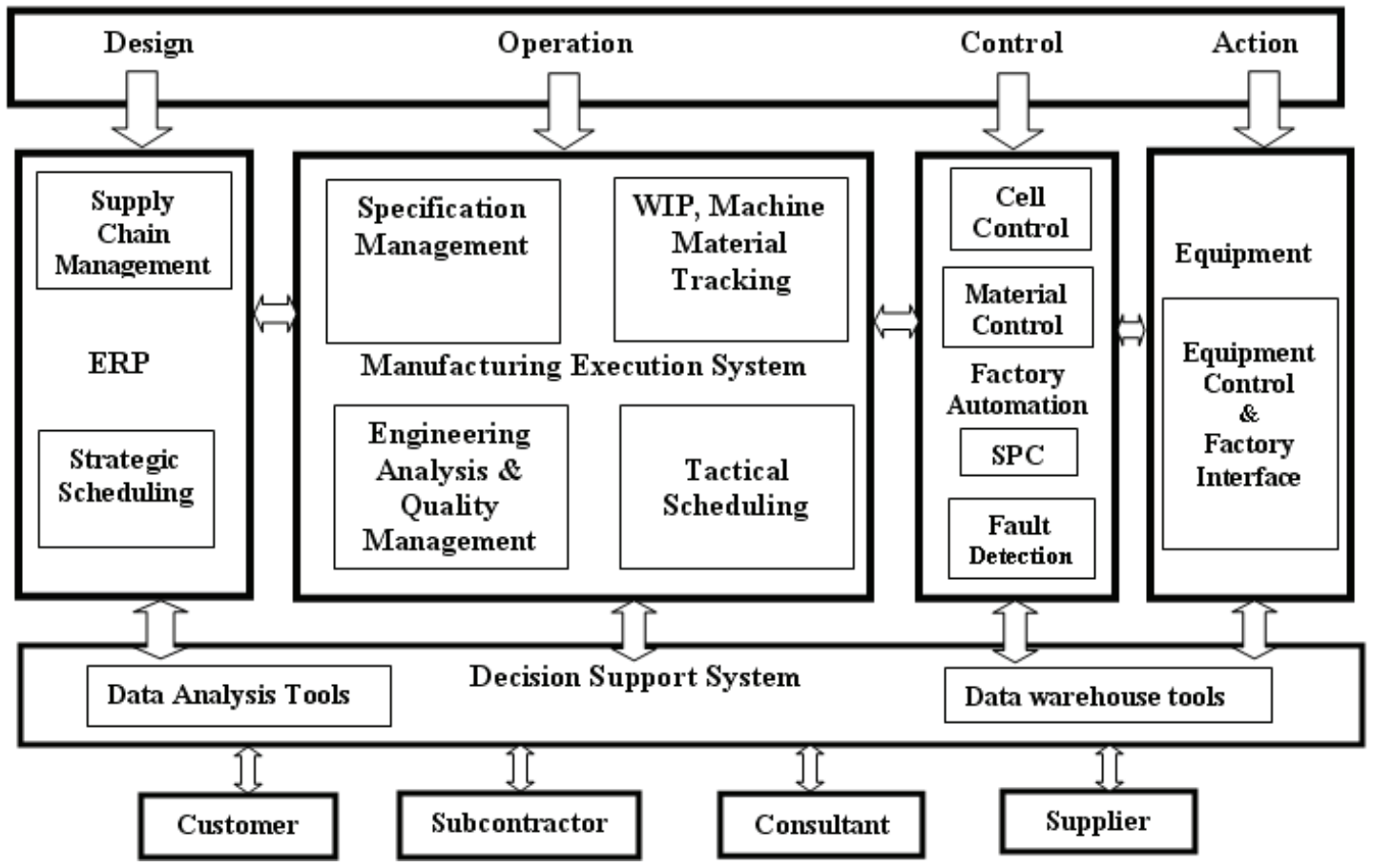

Figure 1 Integrated Manufacturing System Components

\section{DISTRIBUTED MANUFACTURING SYSTEM MODEL}

For an integrated system be effective throughout a network of distributed manufacturing system, it must be connected to Internet (or any world-wide information area network).The e-manufacturing system would include a mixture of both traditional functional systems and newly transformed network-based components as shown in Figure 2.

In this paper we also focus on applications that concentrate on quality control for product via internet based system. Designing a quality strategy to handle data analysis and reporting processes is a vital activity for manufacturing systems. 


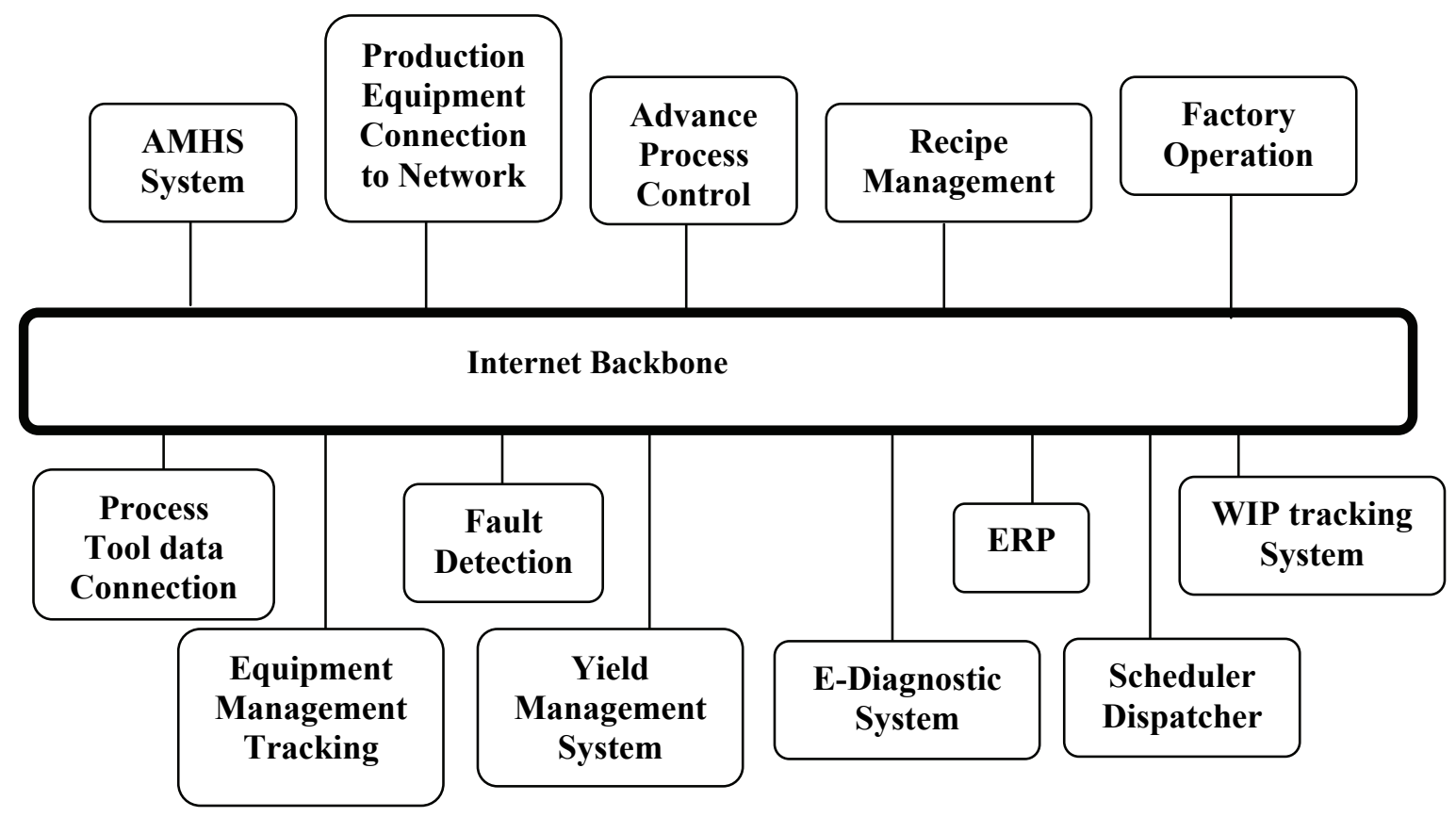

Figure 2 Conceptual Model of e-Manufacturing

\section{STATISTICAL E-BASED QUALITY CONTROL FOR DISTRIBUTED MAUFACTURING SYSTEM}

In general, an internet based manufacturing system must consist of all process of figures 1 and 2 over internet. Starting with the non machined stage, the work-pieces are processed in the sub-processes until the final product is obtained. In each sub-process the work-piece quality must be measured. This system must be connected to Internet (or any world-wide information area network) according to Figure3.

For designing e-based quality controller, measurements of process $k$ must be captured form shop floor via internet, and after processing in controller, appropriate policy must be applied. It usually depends on the process knowledge and experience of the process operator to adjust the parameters of the machine manually. If errors are observed at the end of a sub process, the failure mode and effects analysis (FMEA) is able to list up possible reasons of the failure and makes suggestions for an improvement. The statistical process control (SPC) investigates the measured data with statistical methods in order to predict development in the sub process in the near future. But, both methods are not able to define an appropriate control action to optimize the work piece 
quality immediately and automatically in a closed loop. It is the idea of the model based quality control to use a mathematical model of the process plant for the interpretation of the measured data (Sawodny and Goch, 1997). The control unit has to determine its output such that in the model the measured failure is compensated as good as possible. Designing a quality strategy to handle data analysis and reporting processes is a vital activity that advanced manufacturing systems need to perform for enterprises to survive in the increasing competitive global market.

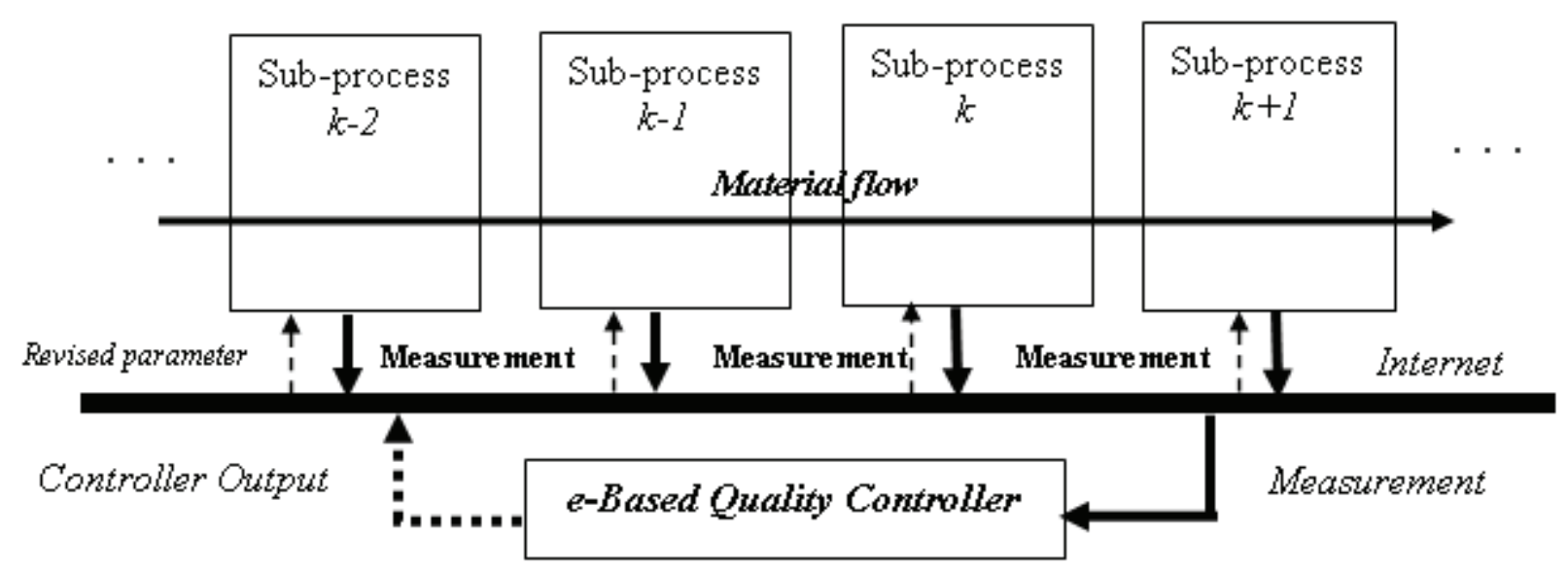

Figure 3 Conceptual model of internet based manufacturing system

Quality engineers and managers have been dependent on quality information system (QIS) to access data such. Quality managers have used quality control systems such as statistical process control, production part approval process, failure mode effects analysis, gage calibration and document control. Being mostly stand-alone applications these individual applications have could not meet quality objectives required for today's complex manufacturing processes. Their inability to provide effective links to enterprise integrated management systems has become a major drawback to be used effectively in a distributed organization. For example, many computer based automation software products in the past were incapable of interfacing with other industrial automation systems. Failing to use standard protocols for communication and lacking the technology for seamless connections to the corporate database, these software systems turned into islands of information and lost much of their designed benefits and savings. 
Any internet based system architecture is structured around a three-layered framework: information, application and end-user layer (Rodriguez and Al-Ashaab, 2005).The end- user layer is connected to the application web server (application layer), which in turn is connected to the information databases (information layer). The application layer consists of two elements: decision support applications and information management tools. The web-based interface of the end-user layer helps users to view and use different decision support applications and tools. The product data is produced and used by different engineering applications throughout the entire production processes. The data is usually stored in what is called a product model. The structure of a product model is related to the type of engineering application that it supports.

The factory-floor automation systems provide native links to the administration so that mission-critical data collected from the factory-floor are captured and stored. Manufacturers currently measure process and performance to improve quality in production. Today, many companies are committed to extending the application of quality measures to product design, customer service, finance, and procurement. Sometimes such effort further extended to facilities in different geographic locations as well as to suppliers, strategic partners, and sales channels. Statistical e-based quality control enables continuous quality improvement through easy and cost-effective access to quality data. It requires an internet-enabled software solution to allow individuals to access the quality data for various analyses. A statistical internet-based quality control also needs a dynamic web-based application that serves as a quality portal, so that users can easily and cost-effectively integrate a wide array of information for quality improvement. From within a web browser, authorized users should be able to analyze their operations real-time. It must be configurable to meet a wide range of needs for information and in-depth quality analysis. Statistical internet-based quality control must have a continuous quality improvement strategy to ensure quality products and services. Empowering individuals with quality analysis and reporting capabilities shifts the responsibility for quality beyond the hands of a few individuals or a specific division to the whole organization. Everyone in the organization takes on the responsibility to continuously assess, improve, and monitor quality. The quality control system must meet the technological requirements of client/server architecture, Structured Query Language (SQL) compatibility, interoperability with other automation systems, and data exchange standards such as OLE automation and OLE for process control. In addition, by linking to the enterprise database, an SPC system eliminates redundant data entry and resulting data 
errors. Using OLE automation or OPC, the e-based system automatically creates part or process specifications from design models stored in other applications. Implementing an enterprise wide quality control system will significantly reduce overall cost of ownership compared to stand-alone or departmental-based software. An organization operating multiple locations on a single server can benefit also from the economy of scale.

As shown in Figure4, for implementing such a system we need a Client/server architecture that allow the SQL database (e-server) to be connected to the quality control database in information layer. Statistical e-based QC utilizes statistical e-server to perform all data access, transformation, analyses and output creation. Users request an action from within the GUI in a browser (the presentation layer) by hitting a button, selecting a menu or completing a form. Once the request is received by the presentation layer, the presentation layer (Active Server Pages) handles the request and directs it to the application (Business Logic Application Layer) for processing. The application processes the request and determines appropriate actions needed to complete the request via interaction between e-based QC application and e-server. For computation, analysis and graphics, the application analyzes data with the help of database to response the request via ODBC tools. After requests directed to this platform, computations performed and results return in the form of HTML table or data file to the reporting tools. (i.e. accept data from the application, process the request, generating an SPC chart, and return the resulting graph in the form of image file or HTML or sample the data, check SPC rules and make alarm by sending rule violation information to the application).

e-Server is a module used for statistical analysis by comparing product key attribute $i$ by $\operatorname{Ref}^{k}(i)$ which comes from design specification. All processes of manufacturing system can be parameterized for key attribute $i$ by measurements $M^{k l}{ }_{t}(i)$ and control output $C^{k l}{ }_{t}(i)$ at time $t$ in shop floor $l$ for process $k$.

In addition to highly customizable SPC charting capabilities, e-Server should have the capacity to provide an extensive suite of graphics and statistical analysis methods as well as the dynamic reporting capability via internet, intranet or extranet so as to support continuous process improvement. It should also be able to perform real-time display of quality control charts to monitor processes. It should be a flexible, full-featured analysis required for continuous quality improvement. Data preprocessing procedure for customer analysis tracks various online activities of customers. This involves capturing information about individual user, constructing logical user "session", and creating key attributes or variables. 


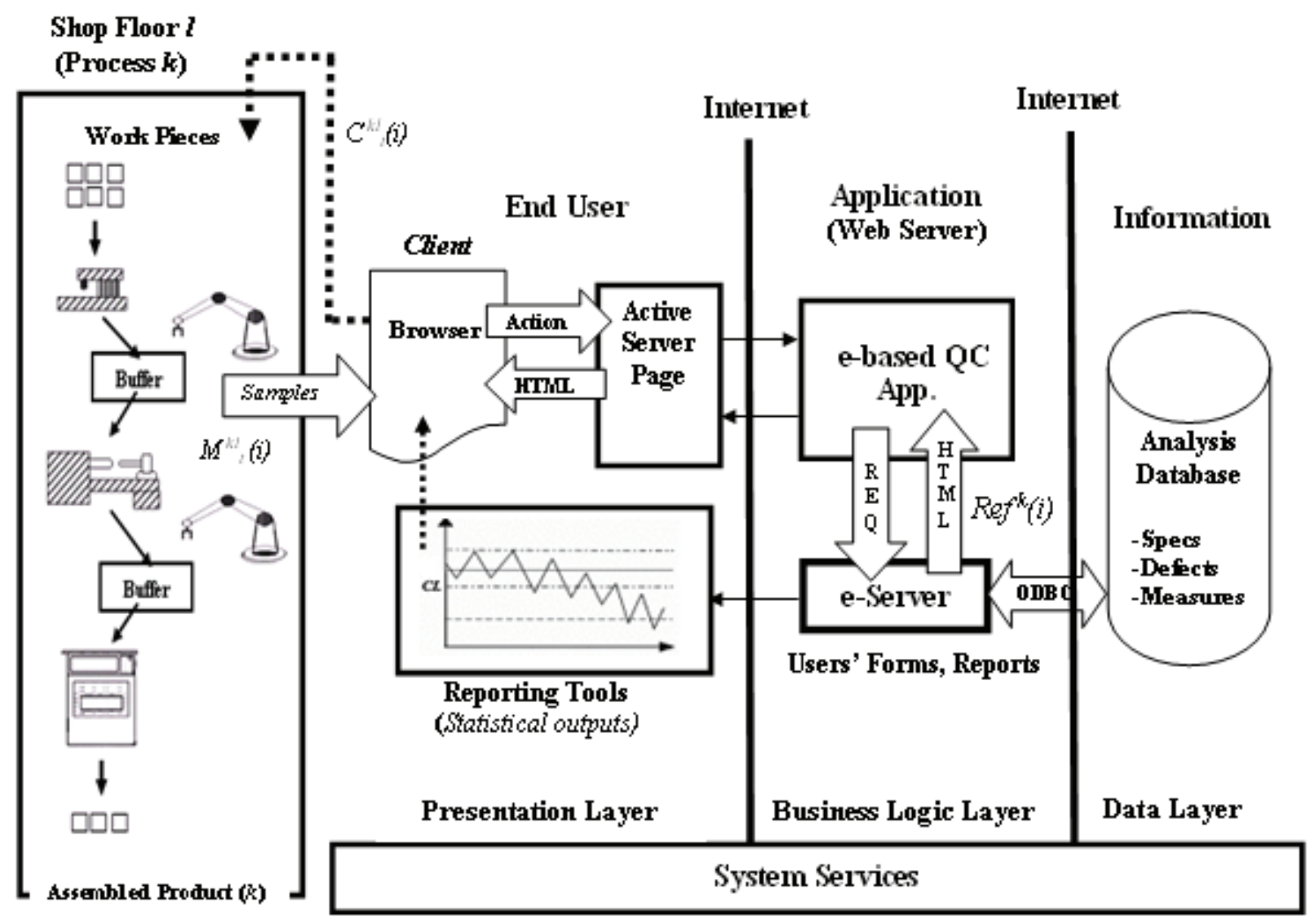

Figure 4 Components of e-based Quality Contorl

\section{MATHEMATICAL FOUNDATION FOR E-BASED QUALITY CONTROL}

To implement a mathematical model of e-QC layer, we present internal blocks of ebased quality control module as shown in Figure5.

In order to supply the controller itself with the complete information about the current process state, a detector unit is introduced which receives the measurement $M{ }^{k l}{ }_{t}(i)$ for frequency of times, and passes the state information $S^{k l}{ }_{t}(i)$ to the controller. The measurement at the end of each sub process is received only in the step of the workpieces that are passing through and the control output is determined only once for each work-piece. 


\section{Notations:}

$w_{i} \quad$ attribute $\mathrm{i}$

$M^{k l}{ }_{t}(i) \quad$ measurements vector at time $t$ in shop floor $l$ for process $k$ on attribute $i$

$S{ }^{k l}{ }_{t}(i) \quad$ state information at time $t$ in shop floor $l$ for process $k$ on attribute $i$

$\operatorname{Ref}^{k}$ (i) reference value for process (product) $k$ on attributes $i$

$f^{{ }^{k} j l} \quad$ frequency of quality key attribute $i$ in product $j$ at location $l$ for process $k$

$L S L^{k}(i) \quad$ lower specification limit for process $k$ on attribute $i$

$U S L^{k}(i) \quad$ upper specification limit for process $k$ on attribute $i$

$L C L^{k} \quad$ lower control limit for process $k$

$C L^{k} \quad$ central control limit for process $k$

$U C L^{k} \quad$ upper control limit for process $k$

$\delta_{k} \quad$ standard deviation for process $k$

$m \quad$ dimension of key attribute

$p \quad$ the number of locations

$T \quad$ time period duration

$C^{k l}{ }_{t}(i) \quad$ control chart vector $\left[L C L^{k}, C L^{k}, U C L^{k}\right]$ at time $t$ in shop floor $l$ for process $k$ on attribute $i$

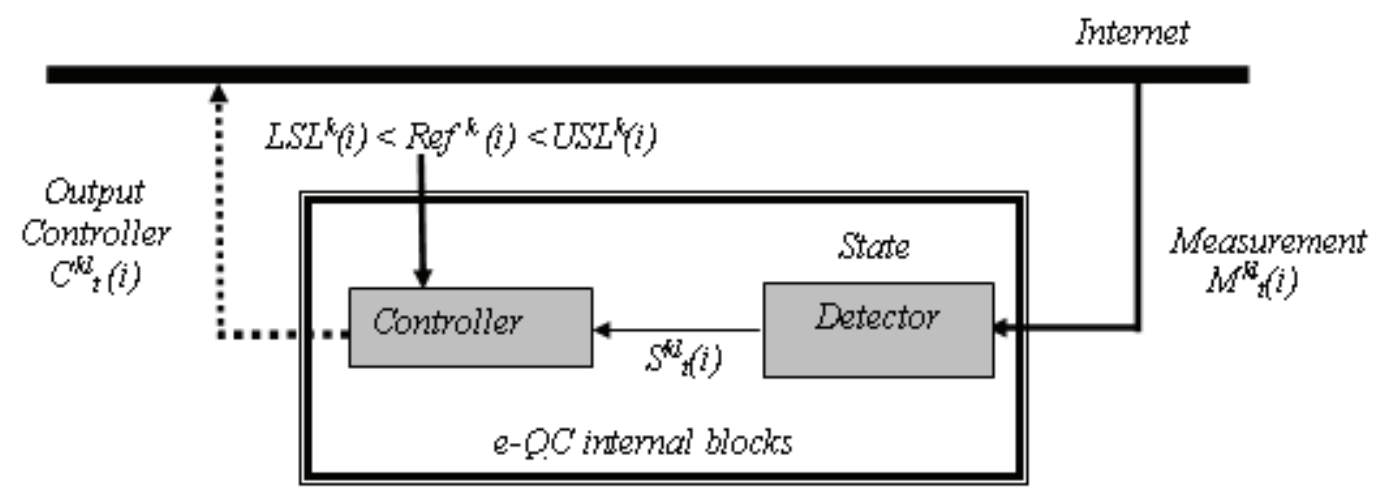

Figure 5 Internal blocks of e-based quality controller

The control problem is now to find a controller output $C{ }_{t}^{k l}(i)$. In general, this may need a sequence steps until Ref ${ }^{k}(i)$ could finally be reached. A process is called controllable in one step for any given state $S{ }_{t}^{k l}(i)$, if the measurement $M{ }^{k l}(i)$ can reach 
the reference value $\operatorname{Ref}^{k}(i)$ :

$$
\operatorname{Lim}\left|\operatorname{Ref}^{k}(i)-M^{k l}{ }_{t}(i)\right| \rightarrow 0
$$

As a result of tracking the quality of each product, we obtain the vector of comprehensive key attribute on quality; $M^{k l}{ }_{t}(i)=\left[w_{1}, w_{2}, \ldots, w_{m}\right]^{n}$. The quality information is collected through electronic customer interaction on the web. As for quality key attribute, we use following formulations for control chart vector:

$$
C L^{k}=\frac{\sum_{t=1}^{T} \sum_{i=1}^{m} \sum_{l=1}^{p}}{T \cdot m \cdot p}\left(\frac{f^{k}{ }_{i j l}}{\sum_{i=1}^{m} f^{k}{ }_{i j l}} \times \frac{f^{k}{ }_{i j l}}{\sum_{j=1}^{n} f^{k}{ }_{i j l}} \times \frac{f^{k}{ }_{i j l}}{\sum_{l=1}^{p} f^{k}{ }_{i j l}}\right)=\frac{\sum_{t=1}^{T} \sum_{i=1}^{m} \sum_{l=1}^{p}}{T \cdot m \cdot p}\left(\frac{\left(f^{k}{ }_{i j l}\right)^{3}}{\sum_{i=1}^{m} f^{k}{ }_{i j l} \sum_{j=1}^{n} f^{k}{ }_{i j l} \sum_{l=1}^{p} f^{k}{ }_{i j l}}\right)
$$

To reach this state we compute from array $M^{k l}{ }_{t}(i)$ :

$$
\begin{aligned}
U C L^{k} & =C L^{k}+3 \sigma_{k} \\
L C L^{k} & =C L^{k}-3 \sigma_{k}
\end{aligned}
$$

That $\sigma_{k}$ results from process standard deviation (calculated according to samples). After determining that process is out of control the controller applies appropriate output value:

$$
C_{t}^{k l}(i)=\left[L C L^{k}, C L^{k}, U C L^{k}\right]
$$

These reports are drawn graphically by e-Server on internet browser of shop floor and quality engineer could decide appropriate policy according to the observed control charts.

\section{Numerical Illustration}

For example, consider a company has two shop floors that are located in two places for producing of three types of products. The measurement of $M^{k l}{ }_{t}(i)$ has been obtained for duration of $T=30$ with frequency of quality key attributes value between (0-100) as shown in Figure 6. According to the control chart we can obtain $C^{k l}{ }_{t}(i)$ and then we could monitor for the process control and develop the QC charts with appropriate policy and applying evolutionary algorithm for online changes in attributes. 


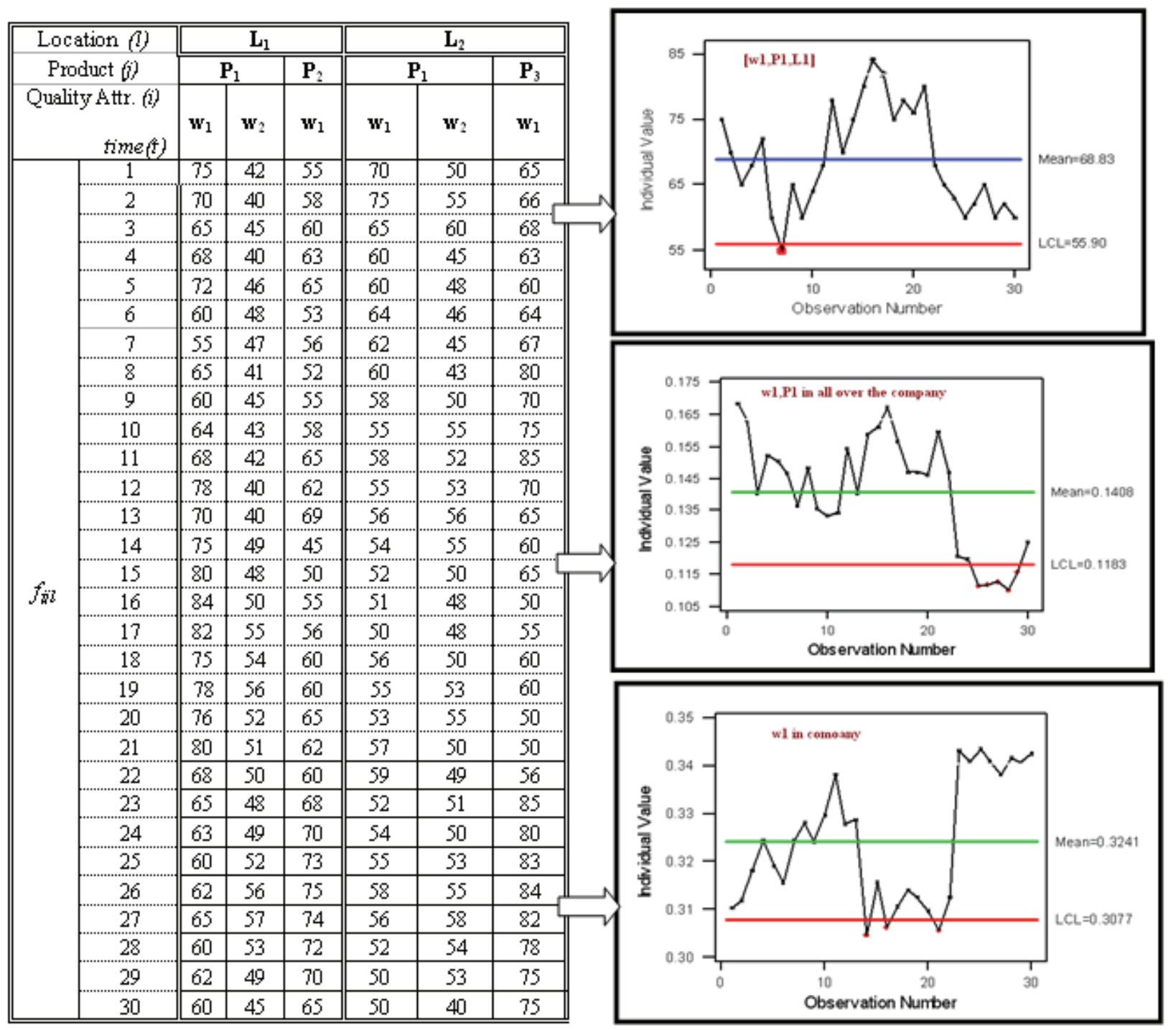

Figure $6 M^{k l}{ }_{t}(i)$ vector and corresponding control charts

\section{Distributed Quality Profile Using Evolutionary Algorithm}

Quality management for an enterprise wide e-based QC system focuses on analyzing data to make decisions that affect future production and revenue. The analysis includes real-time enterprise wide view of operations levels, and batch report according to predetermined time period. We can apply the evolutionary rule to automatically modify the structure of e-based quality information. It updates the quality profile in a dynamic fashion. We suggest the following steps as an evolutionary algorithm. 
Step0. $T=0$.

Step1. Obtain the quality information profile using e-QC model.

Step2. Apply distributed quality management and quality decision making policy to modify quality structure.

Step3. Implement feasibility analyzer for quality decision making.

Step4. $T=T+1$.

Step5. Update the e-based quality network profile and go to step1.

As time passes new attributes in the quality information space profile can be generated. In the newly generated profiles, some attributes may take a value beyond the lower or upper bound of the range of variables appropriate for quality decision making. Then we use feasibility analyzer to check the feasibility boundary of attributes. New attributes are collected and stored to form a meta-decision support.

\section{CONCLUSIONS}

Word-wide competition leads to the need for new systems of control for DMS. The integration of information systems and self-organization factors provide us with opportunities to fulfill such needs to adapt quickly to the changes in environment. The new control systems should be the integration of new technologies, tools and paradigms. The emergent control architecture relies mainly on the multi-agent technology to support the autonomous and adaptive control. A multi-agent system is suitable especially to the DMS, which presents modular, decentralized, changeable, ill-structured and complex issues. To deal with disturbances that deviates the process from the original plans, the system should respond dynamically and quickly using a mechanism to find out the best plan to handle the disturbance based on pre-defined rules and knowledge acquired through past experience. In this paper, we introduced a framework of e-based quality management and developed a new formulation that would provide a web-based solution for real time process. The present logic is applicable for using a wide variety of quality characteristics as key attribute. As a result of using statistical e-based quality profile, anyone in an enterprise can contribute to quality improvement efforts. Web-enabled quality control system will present an extensive connectivity outside a plant. A customer's engineer could tour the plant site and check the profile online. It is used mostly within a factory today, but after some successes, and with the process in place, access to quality data will be extended across the supply chain and to customers. One 
should be able to obtain information about the batch he ordered and see how it conforms to specifications. Based on the research and framework done in the distributed manufacturing systems we developed an analytical approach on e-based quality control. For a customer interface and reliable and sustainable information provision, an evolutionary rule is suggested to create a dynamic quality profile. A mathematical model for an e-based statistical control on the basis of e-based quality profile can be elaborated in future research.

\section{REFERENCES}

Anderson, J., Rungtusanatham, M., \& Schroeder, R.G. (1994). A theory of total quality management underlying the Deming management method. Acad. of Manage. Rev.3, 472-509.

Dean, J.W., \& Snell, S.A. (1991). Integrated manufacturing and job design: moderating effects of organizational inertia. Acad. of Manage. J. 34, 776-804.

Flynn, B.B., \& Flynn, E.J. (2005). Synergies between supply chain management and quality management: emerging implications. International Journal of Production research, Vol 43(16), 3421-3436.

Flynn, B.B., Sakakibara, S., \& Schroeder, R.G. (1995). Relationship between JIT and TQM: Practices and Performance. Academy of Management Journal, Vol 38(5), 1325-1360.

Hao, Q., Shen, W., \& Wang, L.,(2005). Towards a cooperative distributed manufacturing management framework. Computers in Industry. Vol 56, 71-84.

Jagdev, H.S.,\& Thoben, K.D. (2001). Anatomy of enterprise collaboration. Production Planning and Control, Vol 12(5), 437-451.

Leitao, P., \& Restivo, F. (2000). A framework for Distributed Manufacturing Applications. Proceedings of ASI'2000 International Conference, Bordeaux, France.

Mehra, S., Hoffman, J.M., \& Sirias, P.,(2001). TQM as a management strategy for the next millennia. Int. J. Op. \& Prod. Manage, Vol 21(5/6),855-876.

Molina, A., Al-Ashaab, A., Ellis, T., \& Young, R. (1995). A review of computer-aided simultaneous engineering systems. Research in Engineering Design, Vol 7, 38-63.

Rodriguez, K., \& Al-Ashaab, A. (2002). A review of internet based collaborative product development systems. Proceedings of the International Conference on Concurrent Engineering: Research and Applications, Canfield, UK.

Rodriguez, K. \& Al-Ashaab, A.(2005). Knowledge web-based system architecture for 
collaborative product development. Computers in Industry, Vol 56, 125-140.

Sawodny, O., \& Goch, G. (1997), Design of Post-Process Quality-Control Loop for the Turning Process. Proc. of the IFAC Workshop on Manufacturing Systems „IFACMIN '97: Modelling, Management and Control, 279-284

Su, D., Ji, S., Li, J., \& Chen, X. (2002). Web-enabled collaborative environment for integrated design and manufacture. Proceedings of the International Conference on Concurrent Engineering: Research and Applications, Canfield, UK. 
Contemporary Management Research 118 